\title{
A Few Spanish Pearls for the American Journal of Gastroenterology
}

Angel Lanas, $\mathrm{MD}, \mathrm{PhD}^{1,2,3}$

Am J Gastroenterol advance online publication, 7 November 2017; doi:10.1038/ajg.2017.410

In the Red Section of this issue of the American Journal of Gastroenterology, well-known specialists in their respective fields present highlights from gastroenterology research in Spain. These reports illustrate how well-organized network collaborations could impact the scientific literature worldwide, as these collaborations are positioned at the frontier of knowledge and could reveal innovative approaches to the diagnosis and treatment of patients with digestive diseases. These achievements are especially notable, given that they occurred during a serious worldwide economic crisis that affected research in many countries, especially those that do not generally prioritize science $(1,2)$.

Most of the successes presented here were obtained under the umbrella of two national organizations. One is the CIBERehd, an acronym of the Spanish name "Centro de Investigation Biomédica en Red para Enfermedades Hepáticas y Digestivas" or "Biomedical Research Networking Center Consortium in Hepatic and Digestive Diseases" in English. The CIBERehd (http://www.ciberehd. org) is part of a larger entity known as "CIBER", which has additional areas of interest focused on a variety of human diseases. The CIBER was initiated and organized by the National Institute of Health Carlos III (http://www.eng.isciii.es), in charge of coordinating Spanish biomedical research. The CIBERehd is a "virtual" center, combining the efforts of the best research groups in gastroenterology and hepatology across the country, and it funds, awards, and prioritizes collaborative research among the groups.

The second organization may have even greater merit in this context, and is known as the Asociación Española de Gastroenterología (AEG) (http://www.aegastro.es), also known as the "Spanish Association of Gastroenterology" in English. The AEG has promoted important network research collaborations joining the efforts of many clinical research groups of different sizes and capacities. Many of these groups are not yet included in the CIBERehd, which limits their capacity for funding. The AEG has several working groups (Esophagus-Stomach-Duodenum, GI Oncology, Endoscopy, Neuro-gastroenterology and Motility, Inflammatory Bowel Diseases, Pancreas and Biliary Tree) and platforms and collaborates closely with other scientific organizations, including the Spanish Working Group on Crohn's Disease and Ulcerative Colitis
(GETECCU) with its registries and methodological unit, as well as the Spanish Pancreatic Club. In just 10 years, CIBER investigators have published $>7200$ articles in the top medical journals, and almost 2600 of them were related to CIBERehd. The AEG groups have published almost 300 studies over the same period, fifty of which have been published in The American Journal of Gastroenterology $(3,4)$.

One of the five reports published in this special section illustrates two of the tools enabling this small miracle, the ENEIDA (Estudio Nacional en Enfermedad Inflamatoria intestinal sobre Determinantes genéticos y Ambientales) and the AEG-REDCap platforms. Adrian G. McNicholl and Javier P. Gisbert describe how "cost-zero" research in Spain combined with international competitive pressure created a strong collaboration of independent support networks through researcher associations and societies, and how this collaboration has become one of the most important contributors to Spanish Gastroenterology. ENEIDA, created by GETECCU, is a nationwide, hospital-based registry and biobank of Inflammatory Bowel Disease patients from 100 Spanish hospitals. This database provides an infrastructure for the development of multicenter studies of all types. The impact and success of ENEIDA has grown exponentially and has become an inspiration for other European organizations replicating this model. The other major platform is the AEG-REDCap, created by AEG within a consortium agreement with Vanderbilt University (5). AEG-REDCap provides scientific methodological support and online data capture/management tools to over 50 projects, including more than 200 databases and 1300 researchers. The success of this initiative inspired participation by many European researchers in this local Spanish platform. The European Registry on Helicobacter pylori management is the best example of this success as well as the largest international project, with over 25,000 cases already registered.

In the pages that follow, Enrique Quintero and Antonio GimenoGarcía offer a perspective on colorectal cancer screening based on studies conducted in Europe in general, and Spain in particular. Much of that knowledge was again obtained within the collaborative network of the GI Oncology group of AEG. This AEG working group has efficiently and successfully generated ongoing projects,

${ }^{1}$ Service of Digestive Diseases, University Clinic Hospital Lozano Blesa, University of Zaragoza, Zargoza, Spain; ${ }^{2}$ Instituto de Investigación Sanitaria Aragón, Zargoza, Spain; ${ }^{3}$ Centro de Investigation Biomédica en Red para Enfermedades Hepáticas y Digestivas (CIBERehd), Madrid, Spain. Correspondence: Angel Lanas, MD, PhD, Servicio de Aparato Digestivo, Hospital Clínico Universitario, C/ San Juan Bosco 15, Zaragoza 50009, Spain. E-mail: alanas@unizar.es 
including the EPICOLON (estudio de la EPIdemiologia del cáncer de COLON) I and II as well as COLONPREV (COLON PREVention study), which have already resulted in important reports. The COLONPREV project (6) will be the first RCT to provide 10-year mortality data, comparing colonoscopy and biennial FIT, as screening methods for intermediate risk populations over the age of 50 . On the basis of the local and regional studies summarized in their report, Quintero and Gimeno-García conclude that under conditions of universal access to our National public Health System and coming from a Spanish perspective, the current recommendations for colorectal cancer screening of first-degree relatives of patients with colorectal cancer should be revised for individuals with only one index case in the family. In their view, the evidence currently supports that these patients should follow a similar screening strategy as those classified within the average-risk population, and that fecal immunological tests (FIT) should be offered as an alternative to colonoscopy screening in this population.

Enrique de Madaria reports another exciting outcome of network collaboration for the study of pancreatic diseases in Spain. He presents four clinical multicenter studies based on basic research addressing the effect of statins in experimental pancreatitis, and suggests a potential role for these drugs in improving the disease course as well as several outcomes. The EST-CPRE study, a retrospective analysis of consecutive patients undergoing Endoscopic Retrograde Cholangiopancreatography (ERCP) in four centers in Spain, found that patients consuming statins had a $70 \%$ lower risk of post-ERCP acute pancreatitis. This result prompted the initiation of a pan-European prospective cohort study involving centers from Spain, Italy, Finland, Croatia, Sweden, and Romania to confirm these results with the STARK study (STAtins and RisK of post-ERCP acute pancreatitis). The STAR-TREC trial is another collaborative study, involving centers from Spain, Peru, and Bolivia, aiming to investigate whether atorvastatin will be useful in the prevention of acute pancreatitis after ERCP. Finally, Madaria presents the SIMBA trial, (SIMvastatin in the prevention of recurrent acute pancreatitis, a triple-Blind, rAndomized controlled trial) designed to investigate the effectiveness of simvastatin ( $40 \mathrm{mg}$ daily) in decreasing the incidence of new episodes of $\mathrm{AP}$ in recurrent cases. This multicenter, randomized, triple-blind, placebo-controlled trial is focused on adult patients with at least 2 episodes of AP. Fifteen centers from Spain have joined SIMBA, and recruitment has already started (7).

The European and especially the Spanish contribution to our understanding of Eosinofilic Esophagitis (EoE), a relatively new diagnosis, has been notable over the last 5 years. Javier Molina and Alfredo Lucendo are two of the most important researchers in this area (8). Their studies have been conducted in Spain, and are now being projected within the United European Gastroenterology (UEG) organization. Crucially, these studies have advanced the understanding that GERD and EoE are not mutually exclusive disorders, and that there is a clear EoE phenotype with complete remission on PPIs, with or without GERD. European studies have demonstrated that phenotypic, molecular, and mechanistic features cannot distinguish between patients with EoE that do or do not respond to PPI. Therefore, Molina and Lucendo conclude that
PPI therapy responders should be included within the spectrum of EoE, suggesting novel questions requiring investigation with new compounds such as vonoprazan, the first agent of a new class of potassium-competitive acid blockers.

The last report comes from one of our most relevant investigators on functional disorders. Fermin Mearín provides a provocatively titled report on the specifically Spanish view of IBS. Fermín Mearin has conducted several studies within the AEG working group, and is one of the researchers most familiar with the perception of this disease by Spanish and European gastroenterologists (9). Despite living in a Mediterranean country and consuming a Mediterranean diet, Spanish people are not "immune" to IBS. We appear to have similar IBS subtypes as patients from other parts of the world, with small differences relative to Northern European Countries and the USA. Mearin also points out that Spanish IBS patients seek medical attention more frequently than patients from other countries. This may be due to our public and open access universal health systems, and could have a tremendous impact on costs. Clearly, the regional IBS perspective described by Dr. Mearin will be crucial for an improved understanding of this disease, and will open areas of research for those interested in functional disorders.

These are just some examples of the excellent research currently performed in Spain, and reflected the experiences of hardworking doctors and scientists in harsh economic environments. One could imagine what they would accomplish under a better support from our national and regional institutions.

\section{CONFLICT OF INTEREST}

Guarantor of article: Angel Lanas, MD, PhD.

Specific author contributions: Angel Lanas wrote the manuscript. Financial support: This report was partially funded by a grant from "Instituto de Salud Carlos III (ISCIII) (PI14/01218).

Competing interests: None.

\section{REFERENCES}

1. Pain E. European debt crisis. Research cuts will cause 'exodus' from Spain. Science 2012;336:139-40.

2. Torjesen I. Funding crisis forces Cancer Research UK to cut spending by 10\%. BMJ 2011;343:d8068.

3. Lanas A, García-Rodríguez LA, Arroyo MT et al. Effect of antisecretory drugs and nitrates on the risk of ulcer bleeding associated with nonsteroidal anti-inflammatory drugs, antiplatelet agents, and anticoagulants. Am J Gastroenterol 2007;102:507-15.

4. Gisbert JP, Calvet X, Cosme A et al. Long-term follow-up of 1,000 patients cured of Helicobacter pylori infection following an episode of peptic ulcer bleeding. Am J Gastroenterol 2012;107:1197-204.

5. Harris PA, Taylor R, Thielke R et al. Research electronic data capture (REDCap) - a metadata-driven methodology and workflow process for providing translational research informatics support. J Biomed Inform 2009;42:377-81.

6. Quintero E, Castells A, Bujanda L et al. Colonoscopy versus fecal immunochemical testing in colorectal-cancer screening. N Engl J Med 2012;366:697-706

7. SIMBA trial: Simvastatin in the prevention of recurrent acute pancreatitis, a Triple Blind Randomized Controlled Trial EU Clinical Trials Register2016; updated 08-05-2016. Available at https://www.clinicaltrialsregister.eu/ ctr-search/trial/2016-002445-31/ES

8. Lucendo AJ, Molina-Infante J, Arias A et al. Guidelines on eosinophilic esophagitis: evidence-based statements and recommendations for diagnosis and management in children and adults. United Eur Gastroenterol J 2017 2017;5:335-58.

9. Mearin F. Editorial: From the acute infection to the chronic disorder "Don't worry it's just a viral gastroenteritis". Am J Gastroenterol 2012;107:900-1. 